\title{
Impacto de la biblioteca académica mexicana en la sociedad del conocimiento
}

\author{
Impact of the Mexican academic library in the knowledge society
}

\section{Lourdes Quiroa Herrera, Álvaro QuiJano Solís, Guadalupe Vega Díaz, Carolina Palacios Salinas}

1Biblioteca Daniel Cosío Villegas, El Colegio de México. Cuerpo Académico Bibliotecas Académicas, Información y Sociedad. Camino al Ajusco 20 Pedregal de Santa Teresa. México, D.F., C.P., 10740. (1) mquiroa@colmex.mx (2) quijano@colmex.mx (3) guvega@colmex.mx (4) cpalacios@colmex.mx

\section{Resumen}

Se analiza el valor de la biblioteca académica y sus implicaciones en el éxito de las instituciones de educación superior en México. Para ello, considera como marco el documento Value of academic librarie : $A$ comprehensive research review and report de la Association of College \& Research Libraries. Se brinda un panorama general de la sociedad del conocimiento así como del valor de las bibliotecas académicas, se mencionan algunos aspectos sobre su evaluación haciendo especial referencia a la alfabetización informacional y a los servicios y recursos electrónicos de información como los factores clave en el éxito institucional, así como los retos que confrontarán las bibliotecas hacia el final de la década.

Palabras clave: Bibliotecas universitarias. Evaluación de bibliotecas. Alfabetización informacional. Recursos de información electrónicos. Sociedad del Conocimiento. México.

\section{Introducción: las sociedades del conocimiento}

Desde que el concepto de Sociedad del Conocimiento fue propuesto por Drucker en 1969, han surgido otros como "sociedades del aprendizaje" y "educación para todos a lo largo de toda la vida". Por su lado, el de Sociedad de la Información alcanzó una alta popularidad gracias a la trilogía de Manuel Castells (2000) dedicada a la que llamó "era de la información". En todos estos conceptos hay una convergencia sobre la forma de pensar a la nueva sociedad como determinada por una marcada penetración del poder a través de la tecnología, una creciente forma de producción basada en la utilización intensa del conocimiento científico y una transformación del trabajo (UNESCO, 2005).

Hay, también, una idea cada vez más difundida de que la llamada Sociedad de la Información es solo la plataforma inicial necesaria para la construcción de la Sociedad del Conocimiento (SC). Olivé (2009) señala rasgos diferenciado-

\begin{abstract}
This paper explores the value of the Mexican academic library along the lines proposed by the ACRL in its document Value of academic libraries: A comprehensive research review and report. It is a first advance of a broader research on library indicators of the Knowledge Society in Mexico. A general view on the role of the academic library in Mexico emphasizes the information literacy and services and electronic resources as the key factors to evidence the value that the library has for its institution success. Some of the main challenges that will be faced by the academic library in the near future are analyzed within the Mexican context.
\end{abstract}

Keywords: Academic libraries. Library evaluation. Information literacy. Electronic resources. Knowledge Society. México.

res de la SC, como son: el incremento en la creación de información y conocimiento, la capacidad desarrollada para su acumulación y distribución, así como la capacidad social de su aprovechamiento. Idealmente, el motor de la generación de riqueza en esta sociedad es el conocimiento, que se constituye en el factor principal del desarrollo y del bienestar humano. Este enfoque humanista, asigna a los miembros de la SC como principal objetivo el de apropiarse del conocimiento científico, tecnológico y tradicional disponibles, así como utilizarlo de manera autónoma para generar el nuevo conocimiento necesario para comprender y resolver mejor sus problemas.

Para que se lograra lo anterior resultaría indispensable que las políticas públicas fomentaran la creación, el fortalecimiento y la evaluación de sistemas sociales que favorecieran la apropiación, el uso, la generación y la aplicación de la información y el conocimiento para la comprensión y resolución de problemas sociales. Entre estos sistemas están las bibliotecas de todo tipo que, además, deberían incluir entre sus funcio- 
nes la distribución democrática de esa información.

Es sabido que la simple acumulación y disponibilidad de información no generan conocimiento por sí mismas. Mientras la información puede obtenerse cuando la vemos, oímos, memorizamos y guardamos, la apropiación del conocimiento relevante supone habilidades cognitivas, diferenciadas cualitativamente, que requieren un proceso complejo de aprendizaje, para que este conocimiento sea utilizado por el aprendiz e incorporado en su práctica. Sin duda, "Ios procesos de apropiación del conocimiento son mucho más complejos que los de la apropiación de la información" (Echeverría, 2009, p. 44). En efecto, la información se mantiene como un conjunto de datos en tanto no se le trate con discernimiento y espíritu crítico, para analizarla y seleccionar sus distintos elementos e incorporar los que se estimen pertinentes a una base de conocimiento.

El dominio de competencias mentales para transformar la información en conocimiento supone reflexión crítica y aprendizaje teórico, que deberían estar entre los objetivos de la SC. Es este dominio el que nos permitiría orientarnos en lo que conocemos como la explosión de la información. Olivé (2009) señala que una SC debe perseguir que las comunidades y las personas reciban educación y obtengan condiciones que garanticen el desarrollo de sus capacidades para aprovechar el conocimiento existente y generar el que se requiera para la solución de sus problemas, así como que exista una efectiva disponibilidad pública del acervo universal del conocimiento.

\section{Bibliotecas académicas y sociedad del conocimiento}

Entre los sistemas orientados a fomentar la aplicación de conocimiento a problemas de la sociedad, las instituciones de educación superior (IES) son de las gozan de mayor reputación y probada eficacia, de tal forma que las bibliotecas académicas (Bas) de las IES constituyen un elemento importante en el proceso de apropiación de la información para construir la base de conocimiento necesaria para la solución de problemas.

A partir del surgimiento de Internet y, particularmente, de las bibliotecas digitales, las BAs se reposicionaron en el mundo de la información y retomaron un espacio que habían empezado a perder al surgir nuevos formatos de información, particularmente electrónicos. Paradójicamente, Internet es ahora la principal competidora de las bibliotecas académicas, al ofrecer información disponible en texto completo, en millones de fuentes, a lo ancho del mundo y las 24 horas todos los días.

En efecto, el espejismo de un cúmulo de información gratuita y disponible de manera irrestricta ha generado confusión sobre la existencia de la BA como un espacio físico, sustituible por la multiplicación de recursos electrónicos de información y de acceso a éstos, con la idea errónea de suspender acríticamente la compra de materiales impresos.

Por otro lado, se ha acentuado el cambio hacia modelos educativos más participativos y orientados al trabajo colaborativo, que suponen mayores espacios físicos y virtuales para el trabajo en grupo y una mayor interacción entre las comunidades de aprendizaje y los bibliotecarios.

A partir de este entorno, hay una presión cada vez mayor por parte de las instituciones de educación superior para justificar lo que se invierte en la BA (Luther, 2008), lo que implica mostrar evidencias del valor que éstas tienen para sus comunidades (Oakleaf, 2011), al tiempo que también hay necesidad de replantear los modelos de BAs de cara a tendencias como la de los Centros de Recursos para el Aprendizaje y la Investigación -CRAI (1) (véase por ejemplo CRUE, 2005). De cara a estas tendencias, no parecen pertinentes los parámetros que tradicionalmente utilizan las BAs para medir su valor en términos de indicadores cuantitativos asociados con las operaciones convencionales de adquisición de materiales, organización bibliográfica y préstamo, entre otros.

En este orden de ideas, la Association of College \& Research Libraries (ACRL) publicó Value of academic libraries: A comprehensive research review and report, en el cual, a partir de la revisión de la literatura, se proporciona un abordaje distinto del valor de la biblioteca en el contexto de la institución a la que pertenece.

En su documento la ACRL $(2010$, p. 30, 31) señala que a través de la evaluación es posible obtener datos que permitan a los bibliotecarios tomar decisiones e identificar cómo desean alcanzar los propósitos de las IES. Además de contribuir a una mayor credibilidad interna y externa, la evaluación proporciona una oportunidad para la reflexión, la crítica, el aprendizaje organizacional y el posicionamiento institucional (Cfr. Keeling et al. 2008, p. 74, 91).

Se considera que el documento de la ACRL es útil en otros contextos no anglosajones porque constituye un marco para analizar la potenciali- 
dad de las BAs y replantear sus funciones de cara al valor que agregan a sus instituciones.

En este orden de ideas, la investigación Indicadores de impacto de la biblioteca académica en la Sociedad del Conocimiento, de la que este documento es un primer avance, busca producir un conjunto de indicadores diferente de los que se conocen hasta ahora que están más orientados a evaluar la labor intrínseca de las bibliotecas en México. El conjunto de indicadores que se pretende proponer vincula de manera sustancial la labor de las bibliotecas con la producción y transmisión de conocimientos que es la labor fundamental de las IES. La pregunta principal que se plantea es ¿cuál es el impacto de las bibliotecas académicas, más allá de su tamaño y servicios, en la cadena de transmisión de información y de su transformación en conocimiento?

En resumen, el objetivo de la investigación mencionada es proponer un modelo de BA basado en la métrica de los indicadores que permitan caracterizarla de manera cualitativa y cuantitativa, así como evidenciar sus vínculos con la generación de conocimiento en las IES y su transmisión hacia la sociedad del conocimiento.

En este trabajo, que pertenece a la primera etapa de la investigación mencionada, se analiza el tema del valor de las BAs mexicanas de acuerdo con: a) los aspectos relevantes del documento de la ACRL; y b) las implicaciones en la evaluación de la alfabetización informacional, los servicios a la comunidad y los recursos electrónicos para la BA.

\section{El valor de las bibliotecas académicas}

De acuerdo con el documento de la ACRL, es difícil estimar el valor de la biblioteca, aunque se proponen conceptos como retorno de la inversión (RI), basado en los costos y los beneficios obtenidos; así como el uso e impacto de la biblioteca. El documento identifica diez áreas en las que el valor de la BA debe calcularse y medirse. Estas áreas conforman una serie de sectores estratégicos para las instituciones académicas en donde la BA tiene una incidencia directa o indirecta (véase tabla I).

\begin{tabular}{lll}
\hline Enseñanza & Investigación & General \\
\hline Matriculación & Productividad en investigación del profesorado & Prestigio institucional \\
\hline Eficiencia terminal & Fondos para investigación & \\
\hline Éxito laboral de los estudiantes & & \\
\hline Rendimiento de los estudiantes & & \\
\hline Aprendizaje de los estudiantes & & \\
\hline Experiencia de los estudiantes & & \\
\hline Apoyo a la enseñanza &
\end{tabular}

Tabla I. Áreas de impacto de la biblioteca en la misión institucional (traducido de ACRL, 2010, p. 102).

Las áreas detectadas en la tabla I representan factores medibles de éxito institucional en los que la biblioteca ha tenido un impacto, pero que nunca se han evidenciado completamente.

La propuesta de la ACRL tiene sentido para el caso de las BAs en los Estados Unidos de América y considera conceptos que en el caso mexicano han sido poco explorados. Por ejemplo, por razones culturales de aquel país, la biblioteca suele jugar un papel importante para que un estudiante seleccione una institución en particular. En el caso mexicano no se conoce evidencia de que esto ocurra. Otro caso del que se tiene escasa evidencia es el del impacto de la BA en la obtención de fondos de investigación para los profesores. De forma similar al ejemplo anterior, no se conoce con precisión el papel que la biblioteca juega en las decisiones de los comités al asignar fondos a los proyectos presentados.

En el documento de la ACRL se propone que el plan estratégico incluya acciones para evaluar el impacto de la BA en la misión y la visión de las IES. La evaluación podría incluir la medición de indicadores de calidad para aspectos como:

1. Alfabetización informacional (ALFIN).

2. Servicios a la comunidad y recursos de información electrónicos

Debe destacarse que, en la evaluación, los indicadores de calidad sólo tienen significado en relación a la productividad académica y su visibilidad, la docencia y la investigación. Derivado 
de ello, la evaluación requiere de indagar la existencia de correlaciones significativas entre las acciones intrínsecas de la BA y las extrínsecas relacionadas con la misión de la IES.

La ACRL propone el uso de métodos mixtos complementarios y la triangulación de datos con el fin de conectar los indicadores internos y externos. Algunos ejemplos de conexión entre indicadores pueden establecerse a partir de responder preguntas como:¿Qué efecto tienen los programas de ALFIN en la formación del pensamiento crítico de los alumnos? ¿Qué efecto tienen los servicios de referencia en la obtención de fondos para la investigación? ¿Cómo influye el acceso a revistas arbitradas en texto completo en la calidad de los productos académicos?

Como se puede apreciar en los ejemplos anteriores, la conexión entre indicadores requerirá de la obtención de datos de diferentes medios, incluyendo las mediciones indirectas, las evidencias del aprendizaje, los estudios longitudinales, la aplicación de pruebas de rendimiento; así como la realización de estudios de satisfacción y de opinión de los usuarios.

Al final, estos datos tendrían que verse reflejados en los sistemas de evaluación externos, que determinan el ranking de una institución o su acreditación, de tal forma que las instituciones con BAs de vanguardia estén mejor posicionadas y tengan más prestigio. A continuación, se desglosan los aspectos mencionados para la creación de indicadores de calidad en las BAs.

\subsection{Alfabetización informacional}

En la revisión de la literatura que se consigna en el documento de la ACRL se hace énfasis en la evaluación de la alfabetización informacional (ALFIN) como una de las acciones clave para medir el impacto de las BA en las IES. Se persigue el objetivo de que los sujetos posean las competencias para aprender a aprender, para participar de manera informada y para que fortalezcan su pensamiento crítico. Algunos de los aspectos que se integrarían en este sistema de evaluación son:

1. La promoción y el nivel de las competencias en información $(\mathrm{Cl})$ en las IES, la matriculación, retención y la graduación de estudiantes.

2. La integración de los recursos de información en los procesos de enseñanza aprendizaje.

3. La disponibilidad de la información.
4. Los vínculos entre los servicios de información y la preparación de publicaciones, presentaciones o aplicaciones.

5. Los vínculos entre la obtención de fondos y el factor de impacto de la productividad académica (por ejemplo, la citación).

6. La integración de las $\mathrm{Cl}$ en el diseño curricular.

7. La transferencia de las $\mathrm{Cl}$ a diferentes situaciones y contextos académicos.

El documento de la ACRL identifica dos niveles en torno a esta evaluación. El primero es a nivel micro y se centra en la observación de los beneficios y los cambios en los individuos y las comunidades por su participación en actividades, el nuevo conocimiento, el incremento en las habilidades, el cambio en las actitudes o valores y la mejora de las condiciones (Kyrillidou, 2002). Para ello, se requieren instrumentos y técnicas para compilar evidencias, documentos y pruebas confiables; así como el análisis de las discusiones en el aula, las consultas de referencia, las ejecuciones artísticas, los proyectos y las exposiciones de temas (Saunders, 2008, p. 307). Por último, también pueden realizarse estudios longitudinales y de caso para analizar en profundidad los cambios en el comportamiento de los alumnos y las comunidades (Leskes y Wright, 2005, p. 21).

A nivel macro, la valoración de la ALFIN se centra en la aplicación de pruebas con múltiples ítems, como los del Standardized Assessment of Information Literacy Skills y el análisis de las bibliografías (Walsh, 2009, p. 21). Este tipo de valoración podría formar parte de los requisitos de acreditación o del ranking institucional.

\subsection{Servicios a la comunidad \\ y recursos de información electrónicos}

Diversos estudios han demostrado que, actualmente, los usuarios prefieren utilizar la Internet como un recurso de información. Es por ello que, de manera importante, las BA tienen que contar con estrategias de difusión y mercadotecnia para ofrecer servicios que atiendan las actividades académicas de las comunidades a las que sirven, utilizando dispositivos móviles y otras tecnologías de uso común.

En el documento de la ACRL los recursos electrónicos son vistos como ayudas para aumentar la productividad académica y acorde con ello, se han realizado estudios en relación al valor contingente, al valor de retorno de la inversión y de análisis costo-beneficio. 
Como parte de estas valoraciones se encuentran los indicadores estadísticos de uso y de descarga de documentos. Sobre los primeros se ha detectado que no hay uniformidad en cuanto a las métricas que se emplean y que no capturan el por qué o los propósitos de uso (Counter, 2007). Otro tipo de estudios son los de costobeneficio, por ejemplo aquellos que miden la disponibilidad de revistas académicas en el momento en que son solicitadas, en relación con el costo del servicio. Por último, se encuentran los estudios de retorno de la inversión, los cuales consideran el tiempo de retorno del valor, y las ganancias o pérdidas en un año (Luther, 2008 , p. 5). Sin embargo la mejor forma conocida de retorno de la inversión es la obtención de subvenciones para costear los servicios electrónicos y para costear la investigación, por ejemplo, a partir del análisis de las citas y su vínculo con los recursos de información.

\section{La evaluación de bibliotecas académicas en México}

La evaluación de las BAs ha sido un tema de interés por parte de las universidades en Méxi$\mathrm{co}$, desde fines del siglo XX. Al respecto, se han propuesto diversos instrumentos normativos para evaluar a las BAs. Sin embargo, en su mayor parte, éstos son cuantitativos y centrados en la BA. Por ejemplo, la Asociación Nacional de Universidades e Instituciones de Enseñanza Superior (ANUIES) y el Centro Universitario de Investigaciones Bibliotecológicas de la Universidad Nacional Autónoma de México (UNAM) elaboraron el Manual para obtener indicadores como apoyo a la evaluación de los servicios bibliotecarios e instituciones de educación superior (Pagaza, 1989), y el Manual para evaluar la satisfacción de usuarios en bibliotecas de instituciones de enseñanza superior de la República Mexicana (Verdugo, 1989).

En el 2000 se propuso el Modelo para la evaluación integral de las bibliotecas de educación superior de México (Arellano, 2000). En el mismo año se elaboró una Guía metodológica para evaluar las bibliotecas de las instituciones de educación superior de la región centro occidente (ANUIES, 2000).

El Consejo Nacional para Asuntos Bibliotecarios, en el documento Normas para bibliotecas de Instituciones de Educación Superior e Investigación del CONPAB-IES, publicado en el 2005 (p. 29), señala que "la evaluación debe entenderse como un proceso permanente que se realiza mediante la supervisión, verificación, medición y análisis de los recursos y servicios bibliotecarios, así como del resultado e impacto [sic] de éstos en relación con el cumplimiento de las metas institucionales y la satisfacción de los usuarios", lo cual en la práctica no ocurre.

Otro aspecto relevante relacionado con la evaluación son las acciones institucionales dirigidas a la acreditación de las actividades académicas por instituciones extranjeras como la Southern Association of Colleges and Schools (ACS), con normas internacionales como las de calidad ISO 9001; así como las normas mexicanas de acreditación de la ANUIES y las de la Federación de Instituciones Mexicanas Particulares de Educación Superior A.C. (FIMPES). En tales acreditaciones se incluye como uno de sus criterios la evaluación del acervo bibliográfico, el personal y los servicios que brindan las BA.

Además de los instrumentos de evaluación enunciados, es posible señalar algunos estudios de caso en tesis y artículos de difusión. También se han identificado evaluaciones cualitativas (Tarango, Romo, Murguía y Ascencio, 2011) y la aplicación de la metodología LIBQUAL para medir la satisfacción de los usuarios (HerreraViedma, López y Ávila, 2011; Arriola y Butrón, 2011; entre otros). A pesar de los esfuerzos realizados en la evaluación de la $B A$, se considera que en las bibliotecas mexicanas aún se carece de una cultura de la evaluación y de indicadores que reflejen el valor de las BAs en relación con las metas institucionales.

\subsection{Evaluación de la alfabetización} informacional en México

Quizá uno de los aspectos que más ha sido analizado en relación a sus vínculos con las IES es la ALFIN. El tema de la ALFIN, diferenciado del de formación de usuarios en instituciones de educación superior en México, fue por primera vez tratado en el $1^{\text {er }}$ Encuentro Internacional de Desarrollo de Habilidades Informativas (DHI) (1997). Al respecto Lau y Cortés (2000) aplicaron un cuestionario sobre programas de formación de usuarios en universidades, el cual fue contestado por 29 instituciones. De estas, el $82 \%$ dijeron contar con actividades de educación de usuarios, el $56 \%$ tenía un programa formal, sin embargo ninguna reporto actividades de evaluación.

En el IV Encuentro de DHI, realizado en 2004, se identificaron dos trabajos en los que se incluye a la evaluación de los programas como aspectos esenciales. En el primero se hace referencia a los elementos para evaluar programas de DHI (Lau, 2006a) y, en el segundo, se hace una propuesta de evaluación diagnóstica de las competencias en información (Vega, Cortés y Quijano, 2006). 
El V Encuentro de DHI (2006) llevó por título Diagnóstico y evaluación: elementos fundamentales para los programas de Alfabetización Informativa. En este Encuentro se llevaron a cabo dos talleres sobre evaluación de programas de ALFIN y se presentaron varios trabajos entre los que destacan las propuestas metodológicas para la evaluación de programas, incluyendo al docente, los contenidos y el aprendizaje (Lau, 2006b; Aguilar y Cisneros, 2006; Tarango, Ascencio, Murguía y Romo, 2006; Hernández y Terrazas, 2006). También se presentaron experiencias de evaluación de programas (Mears y Cortés, 2006; Almanza, González y Gutiérrez, 2006) y una propuesta de cuestionario para diagnosticar competencias en información (Vega, 2006). Entre los instrumentos para la evaluación del aprendizaje de los alumnos se mencionaron los siguientes: cuestionarios, rúbricas, entrevistas, portafolios y encuestas de opinión. Sobre los elementos a evaluar se mencionaron: acceso, contenidos, manejo documental, competencias en información y aprendizaje. En el VI Encuentro sólo se ubicó un trabajo en relación a la evaluación de las competencias (Tiscareño, 2008).

Un estudio reciente en 60 bibliotecas de IES mexicanas muestra una visión actualizada de los programas de formación de usuarios (Vega, 2011). En el estudio se identificaron 37 programas, de los cuales sólo 12 contaban con un documento escrito. De estos programas sólo ocho incluían actividades de evaluación, los cuales sin embargo se centraban en la valoración del profesor y en los contenidos del programa. A partir de lo anterior se observa la necesidad de vincular estos objetivos con la formalidad que exige la evaluación para, a partir de ello, identificar cuál es la contribución de las bibliotecas académicas en la formación profesional y obtener las evidencias para demostrarlo. Por otro lado, también se hace necesaria la construcción de indicadores de evaluación del aprendizaje y de un uso generalizado de normas de ALFIN.

\subsection{Evaluación de los servicios a la comunidad y de los recursos de información electrónicos}

En México, la mayoría de las BA cuenta con recursos y servicios electrónicos como el catálogo en línea (OPAC), bases de datos, bibliotecas digitales, entre otros, disponibles de manera interna y por acceso remoto. De igual forma, diversas BAs mexicanas han adoptado las herramientas de la Web 2.0 (facebook, sindicación de contenidos -RSS-, blogs, wikis, chats, twitter). También se han desarrollado bibliotecas digitales en México, al respecto Cid (2005) identificó 14 proyectos de bibliotecas digitales, número que ha ido en aumento constante.

A nivel institucional, es posible encontrar tesis de licenciatura y maestría que indagan sobre el uso de los recursos de información, tales como la de Soria (2001) en el Centro de Investigación y Docencia Económica, la de Vélez (2005) en la Facultad de Filosofía y Letras de la UNAM y la de Ortíz (2008) en el Instituto Latinoamericano de la Comunicación Educativa (ILCE). Particularmente, las de Carmona (2007) y Gómez (2011) se centraron en el estudio de uso de recursos de información en alumnos de posgrado.

De manera más sistemática, la Dirección General de Bibliotecas de la UNAM ha realizado diversos estudios sobre el uso de bases de datos. En el artículo Uso de los recursos electrónicos suscritos. Algunos indicadores (2010) se señala que en cuanto a las descargas, los resultados muestran que el uso de bases de datos se ha ido incrementando paulatinamente desde el 2005 (p. 105), con un costo promedio de menos de un dólar por artículo. Otros indicadores que se utilizaron en este estudio fueron los de uso per cápita y la medición de la satisfacción de usuarios. En el caso del uso per cápita se estimaron costos en función del tipo de usuarios (investigador, técnicos académicos, profesores, ayudantes, etc.) y por nivel educativo (licenciatura y posgrado).

Por su parte el Instituto Nacional de Geografía y Estadística (INEGI, 2011) cuenta con un sistema de información sobre ciencia y tecnología, el cual incluye indicadores de patentes solicitadas y concedidas, entre otros. En relación a las actividades de producción científica y tecnológica incluye un apartado en el que se contabilizan los artículos publicados de 1990 al 2009.

Además, el INEGI cuenta con un apartado de sociedad de la información, en el que es posible ubicar el número de usuarios de los servicios de tecnologías de la información a nivel de entidad federativa. Éste comprende las aplicaciones de las tecnologías en los desarrollos de enseñanza-aprendizaje, los cuales en el 2008 eran del $31 \%$ en relación a otras aplicaciones. La tabla II muestra el uso que se hace de la Internet, en donde se destaca la utilización de la Internet para obtener información y para apoyar la educación y la capacitación. 


\begin{tabular}{lrr}
\hline Tipo de uso & Absoluto & $\%$ \\
\hline Usuarios en Internet & 32807240 & 100.00 \\
\hline Para obtener información & 19173742 & 58.4 \\
\hline Para comunicarse & 18837481 & 57.4 \\
\hline Para apoyar la educación o capacitación & 11715805 & 35.7 \\
\hline Para entretenimiento & 9205158 & 28.1 \\
\hline Para operaciones bancarias en línea & 853543 & 2.6 \\
\hline Para interactuar con el gobierno & 384953 & 1.2 \\
\hline Otros usos & 379632 & 1.2 \\
\hline No especificado & 38845 & 0.1 \\
\hline
\end{tabular}

Tabla II. Usos de la Internet (INEGI, Módulo sobre Disponibilidad

y Uso de las Tecnologías de la Información en los Hogares, 2010).

Con relación a la productividad el Sistema Nacional de Investigadores del Consejo Nacional de Ciencia y Tecnología (CONACYT) cuenta con publicaciones como Indicadores de Actividades Científicas y Tecnológicas (2008) y el Informe general del estado de la ciencia y la tecnología (2009), en las que se detallan aspectos relacionados con la producción académica, aunque no se ligan con la actividad bibliotecaria.

Como se puede observar, la información compilada sobre el tema de los recursos electrónicos en México es fragmentada y no permite identificar si están siendo utilizados para apoyar o mejorar los procesos de enseñanza aprendizaje ni tampoco si inciden en la productividad académica.

Un referente indirecto del impacto de la BA es la encuesta de las Mejores universidades que en algunos periodos ha evaluado determinadas bibliotecas académicas (Reforma, 2007). Este estudio tiene una amplia difusión a nivel nacional y es usado para atraer la atención de los alumnos interesados en ingresar a la Universidad.

\section{Reflexiones finales}

Las BAs mexicanas, en su tránsito hacia la SC, deben contar con un plan estratégico con líneas claras de acción que les permita analizar e incorporar las tendencias que se prevén para el futuro. En particular, se requiere promover una mayor interacción con las actividades de docencia e investigación y una mayor presencia de la biblioteca en el proceso de aprendizaje de los estudiantes, lo que puede ocurrir a través de la impartición de cursos y la coparticipación con los docentes en los programas educativos, la administración de cursos en línea, la creación de objetos de aprendizaje y de sitios web, entre otros medios.

Como señala la ACRL (2010), ante los cambios que se observan en el nivel de educación superior, los cambios tecnológicos y el paso hacia entornos virtuales, los bibliotecarios deberán desarrollar diversas habilidades para brindar diversos tipos de servicios virtuales además de los presenciales. Por lo que toca a la educación bibliotecológica estos cambios han demandado una mayor especialización, capacitación continua y la actualización de los programas.

La toma de decisiones que realicen los profesionales de la información en sus gestiones debe considerar el valor de la BA, retomando aquellos aspectos que más convengan a las necesidades que apuntan los documentos de la ACRL. Sería recomendable que esto se haga sin perder de vista los intereses nacionales y locales. En este sentido, se debe identificar cuáles son los intereses en las BA para plasmarlos en sus planes estratégicos que permitan señalar líneas de acción concretas para su adaptación y transformación en un entorno bastante complejo y cambiante.

En México, algunas BAs se han destacado por tener un rol activo en las iniciativas y actividades docentes y de investigación de la institución, como la Biblioteca Daniel Cosío Villegas de EI Colegio de México y el Centro Interactivo de Recursos de Información y Aprendizaje de la Universidad de las Américas Puebla, que proporcionan servicios especializados a través de bibliógrafos que seleccionan recursos y brindan asesoría personalizada a alumnos y profesores, entre otras actividades. Otras instituciones que ofrecen servicios especializados son la Dirección General de Bibliotecas de la Universidad Veracruzana, la Dirección General de Bibliote- 
cas de la UNAM, la Benemérita Universidad Autónoma de Puebla y la red de bibliotecas del Instituto Tecnológico de Estudios Superiores de Monterrey. Sin embargo, no hay estudios que nos lleven a verificar si estos servicios han sido evaluados en relación con su impacto a la comunidad a la que están destinados.

Como se puede observar es mucho lo que todavía se tiene que hacer en relación a los procesos de evaluación en México, principalmente porque se detecta que no hay procesos de planeación y evaluación formales.

\section{Notas}

(1) En la literatura anglosajona se utiliza el término information commons, el cual guarda similitud con el de CRAI (Kranich, 2004).

(2) Las obras señaladas con * fueron citadas en Value of Academic Libraries: A Comprehensive Research Review and Report.

\section{Referencias (2)}

Aguilar, Víctor; Cisneros, Edith (2006). El portafolios como herramienta de evaluación en los programas de DHI. // Mears, Berenice (org.) (2006). Diagnóstico y evaluación: elementos fundamentales para los programas de alfabetización informativa : V Encuentro de Desarrollo de Habilidades Informativas]. Ciudad Juárez, Chihuahua, México. (No publicada) http://bivir.uacj.mx/DHI/5e/Programa Detallado.asp (2011-06-11).

Almanza Morales, José Luis; González Ordaz, Cintia Elizabeth; Gutiérrez Cruz, Víctor (2006). La Alfabetización Informativa en las Humanidades. // Mears, Berenice (org.) (2006). Diagnóstico y evaluación: elementos fundamentales para los programas de alfabetización informativa: $\mathrm{V}$ Encuentro de Desarrollo de Habilidades Informativas]. Ciudad Juárez, Chihuahua, México. (No publicada) http: //bivir.uacj.mx/DHI/5e/ProgramaDetallado.asp (2011-0611).

Arellano Rodríguez, J. Alberto (2000). Modelo para la evaluación integral de las bibliotecas de educación superior de México. México: Secretaría de Educación Pública, Subsecretaría de Educación Superior e Investigación Científica, 2000.

Arriola Navarrete, Óscar; Butrón Yañez, Katya (2011). La cultura bibliotecaria del usuario de bibliotecas académicas: un panorama de su realidad. // Lau, Jesús (coord.) (2011). Satisfacción de usuarios evaluación integral de bibliotecas. Comp. Jesus Lau. Xalapa Ver., Universidad Veracruzana: México: Library Outsourcing Service: Buenos Aires, Argentina: Alfagrama, (ALCl, vol. IV) 2011. 197-222.

Asociación Nacional de Universidades e Instituciones de Enseñanza Superior (ANUIES) (2000). Guía metodológica para evaluar las bibliotecas de las instituciones de educación superior de la región centro occidente. México: Asociación Nacional de Universidades e Instituciones de Educación Superior, Consejo Regional Centro Occidente, Grupo de Trabajo de Bibliotecas, 2000.

Association of College and Research Libraries (ACRL); Oakleaf, Megan (2010). Value of Academic Libraries: A Comprehensive Research Review and Report. Chicago: Association of College and Research Libraries, 2010.http://www.ala.org/ala/mgrps/divs/acrl/issues/value/ val_report.pdf(2011-05-09).
Carmona Victoria, Verónica (2007). Uso de la información en el posgrado medido a través de las tesis de doctorado. México: El autor, 2007. Tesis de maestría, Universidad Nacional Autónoma de México (UNAM).

Castells, Manuel (2000). La era de la información: economía, sociedad y cultura. $2^{\mathrm{a}}$ ed. Traducción de Carmen Martínez Gimeno. Madrid: Siglo Veintiuno, 2000.

Cid Carmona, Víctor (2005). Tendencias y desarrollo de las bibliotecas digitales en México. // Hibay, Denise A. (ed.). Trends and traditions in Latin American and Caribbean history. Papers of the Forty-Seventh Annual Meeting of the Seminar on the acquisition of Latin American library materials. Jun. 1.5, 2002. Albuquerque, N.M. SALALM Secretariat, 2005.

Consejo Nacional de Ciencia y Tecnología (CONACYT) (2008). Indicadores de actividades científicas y tecnológicas: edición de bolsillo. México: CONACYT, 2008. http://www.siicyt.gob.mx/siicyt/docs/contenido/Indicadore s_2008.pdf(2011-04-02).

Consejo Nacional de Ciencia y Tecnología (CONACYT) (2009). Informe general del estado de la ciencia y la tecnología. México 2008. México: CONACYT, 2009. http://www.siicyt.gob.mx/siicyt/docs/contenido/IGECYT_ 2008.pdf(2011-04-02).

Consejo Nacional para Asuntos Bibliotecarios de las Instituciones de Educación Superior (CONPAB-IES) (2005). Normas para bibliotecas de instituciones de educación superior e investigación. Guadalajara, Jalisco, México: Consejo Nacional para Asuntos Bibliotecarios de las Instituciones de Educación Superior, Comisión Permanente de Normatividad 2004-2006. 2005.

Counter. Counting Online Usage of Networked Electronic Resources. 2007. http://www.projectcounter.org/about.ht $\mathrm{ml}(2010: 05: 10)$. $^{*}$

CRUE. Conferencia de Rectores de Universidades Españolas (2005). De las bibliotecas universitarias a los Centros de Recursos para el Aprendizaje y la Investigación. Madrid: CRUE, 2005. http://www.rebiun.org/doc/z2.pdf (2011-05-26)

Drucker, Peter Ferdinand (1969). The age of discontinuity : guidelines to our changing society. New York: Harper \& Row, 1969.

Echeverría, J. (2009). Las repúblicas del conocimiento. // Suárez., R. (coord.) Sociedad del conocimiento: propuestas para una agenda conceptual. México: Universidad Nacional Autónoma de México, Coordinación de Humanidades, 2009. 27-62.

Gómez Restrepo, Alexa Milley (2011). Comportamiento informativo en el uso de las bases de datos bibliográficas de los tesistas en El Colegio de México. México: El autor, 2011. Tesis de maestría, El Colegio de México.

Hernández, Sofía; Terrazas, Fabiola (2006). El Constructivismo: una herramienta de apoyo para el diagnóstico y evaluación de habilidades informativas. // Mears, Berenice (org.) (2006). Diagnóstico y evaluación: elementos fundamentales para los programas de alfabetización informativa. [V Encuentro de Desarrollo de Habilidades Informativas]. Ciudad Juárez, Chihuahua, México. (No publicada.) http://bivir.uacj.mx/DHI/5e/ProgramaDetallado. asp (2011-06-11).

Herrera-Viedma, Enrique; López Gijón, Javier; Ávila, Belén (2011). Satisfacción de usuarios y calidad. El modelo de LibQual y su aplicación en bibliotecas españolas e iberoamericanas. // Lau, Jesús (coord.) (2011). Satisfacción de usuarios evaluación integral de bibliotecas. Xalapa Ver., Universidad Veracruzana; México: Library Outsourcing Service; Buenos Aires, Argentina: Alfagrama, (ALCl, vol. IV) 2011. 111-136.

Instituto Nacional de Estadística y Geografía (INEGI) (2011). Estadística. Ciencia y tecnología. Sociedad de la infor- 
mación. Usuarios. México: INEGI, 2011http://www. inegi.org.mx/Sistemas/temasV2/Default.aspx?s=est\&c=1 9007 (2011-05-26).

Keeling, Richard P.; Andrew F. Wall, Ric Underhile; Gwendolyn J. Dungy (2008). Assessment Reconsidered: institutional effectiveness for student success. International Center for Student Success and Institutional Accountability, 2008.

Kranich, Nancy (2004). The Information Commons: A Public Policy Report. New York: Brennan Center for Justice, Free Expression Policy Project. New York University School of Law, 2004. http://www.fepproject.org/policyre ports/InformationCommons.pdf (2011-05-26). *

Kyrillidou, Martha (2002). From input to output measures to quality and outcome measures, or, from the user in the life of the library to the library in the life of the user. // Journal of Academic Leadership. 28:1: (2002) 42-46.*

Lau, Jesús; Cortés, Jesús (2000). Patron empowerment to use library gateways in lid countries: the case of Mexico. // Lau, Jesús; Cortés, Jesús (comp.). Desarrollo de habilidades informativas en instituciones de educación superior: I Encuentro de Desarrollo de Habilidades Informativas. Ciudad Juárez, Chihuahua: Universidad Autónoma de Ciudad Juárez, 2000. 117-129. http://bivir.uacj. $\mathrm{mx} / \mathrm{dhi} /$ PublicacionesUACJ/Default.htm (2011-05-26).

Lau, Jesús (2006a). Directrices internacionales para desarrollar competencias informativas en los ciudadanos del mundo // Cortés, Jesús; Mears, Berenice (comp.). Implementación de programas de alfabetización informativa: contextos y experiencias: IV Encuentro de Desarrollo de Habilidades Informativas. Ciudad Juárez, Chihuahua, México: Universidad Autónoma de Ciudad Juárez: Library Outsourcing, 2006. 41-57.

Lau, Jesús (2006b). Hastío gratificante: La evaluación DHI para el aprendizaje independiente. // Mears, Berenice (org.) (2006). Diagnóstico y evaluación: elementos fundamentales para los programas de alfabetización informativa: V Encuentro de Desarrollo de Habilidades Informativas. Ciudad Juárez, Chihuahua, México. (No publicada.) http://bivir.uacj.mx/DHI/5e/ProgramaDetallado. asp (2011-06-11).

Leskes, Andrea; Wright, Barbara D. (2005). The art \& science of assessing general education outcomes. Washington, D.C.: Association of American Colleges and Universities, 2005.*

Luther, Judy (2008). University investment in the library: what's the return? A case study at the University of Illinois at Urbana-Champaign. San Diego: Elsevier, 2008.*

Mears, Berenice; Cortés, Jesús (2006). Contexto, protagonistas y evaluación en una materia con valor en créditos: la experiencia en la UACJ. // Mears, Berenice (org.) (2006). Diagnóstico y evaluación: elementos fundamentales para los programas de alfabetización informativa: $V$ Encuentro de Desarrollo de Habilidades Informativas]. Ciudad Juárez, Chihuahua, México. (No publicada). http://bivir.uacj.mx/DHI/5e/ProgramaDetallado.asp (2011-06-11).

Olivé, León (2009). El libro, la lectura y las bibliotecas en la sociedad del conocimiento. // Lectura Vida. 30: 3 (sept 2009) 20-29.

Ortíz Martínez, Claudia Margarita (2008). El uso de las tecnologías de información y comunicación (TIC's) dentro del Instituto Latinoamericano de la Comunicación Educativa (ILCE). México: El autor, 2008. Tesis de licenciatura, Universidad Nacional Autónoma de México (UNAM).

Pagaza García, Rafael (1989). Manual para obtener indicadores como apoyo a la evaluación de servicios bibliotecarios en instituciones de educación superior. Colaborador, Víctor M. Valdovinos Luna. México: Universidad
Nacional Autónoma de México, Centro Universitario de Investigaciones Bibliotecológicas, 1989.

Reforma, El (2007). Las Mejores Universidades 2007-2008. // El Reforma. Suplemento Universitarios (15 de mayo de 2007).

Saunders, Laura (2008). Perspectives on accreditation and information literacy as reflected in the literature of library and information science. // Journal of Academic Librarianship. 34:4 (2008) 305-313.*

Soria Gallegos, Teresita de Jesús (2001). El uso de las nuevas tecnologías de información y comunicación en el Centro de Investigación y Docencia Económicas (CIDE). México: El autor, 2001. Tesis de licenciatura, Universidad Nacional Autónoma de México (UNAM).

Tarango, Javier; Ascencio, Gerardo; Murguía, Patricia; Romo, José (2006). Modelos académicos de las IES y el Desarrollo de Habilidades Informativas: interdependencia en su diagnóstico/evaluación. // Mears, Berenice (org.) (2006). Diagnóstico y evaluación: elementos fundamentales para los programas de alfabetización informativa: V Encuentro de Desarrollo de Habilidades Informativas. Ciudad Juárez, Chihuahua, México. (No publicada). http://bivir.uacj.mx/DHI/5e/ProgramaDetallado. asp (2011-06-11).

Tarango, Javier; Romo, José Refugio; Murguía, Patricia Ascencio, Gerardo (2011). Aplicación de la teoría de los unos para el diagnóstico de satisfacción de usuarios en bibliotecas académicas. // Lau, Jesús (coord.) (2011). Satisfacción de usuarios evaluación integral de bibliotecas. Xalapa Ver., Universidad Veracruzana; México: Library Outsourcing Service; Buenos Aires, Argentina: Alfagrama, (ALCl, vol. IV) 2011, 259-270.

Tiscareño Arroyo, Ma. Lourdes (2008). La evaluación como insumo para la innovación: Curso de Acceso a la Información UACJ. // Mears, Berenice (org.) (2008). La innovación educativa en programas de desarrollo de habilidades informativas: VI Encuentro de Desarrollo de Habilidades Informativas. Ciudad Juárez, Chihuahua, México. (No publicada). http://bivir.uacj.mx/dhi/6e/programa. asp (2011-06-11).

UNESCO (2005). Hacia las sociedades del conocimiento: informe mundial de la Unesco. París: UNESCO. http://unesdoc.unesco.org/images/0014/001419/141908 s.pdf (2011-06-27).

Uso de los recursos electrónicos suscritos. Algunos indicadores (2010). // Biblioteca Universitaria. Revista de la Dirección General de Bibliotecas de la UNAM. 13:1 (enero-junio 2010) 102-112. http://www.revistas.unam. mx/index.php/rbu/article/view/23937(2011-04-10).

Vega, Guadalupe (2006). Diagnóstico de habilidades informativas en alumnos egresados del bachillerato en México. // Mears, Berenice (org.) (2006). Diagnóstico y evaluación: elementos fundamentales para los programas de alfabetización informativa: $V$ Encuentro de Desarrollo de Habilidades Informativas]. Ciudad Juárez, Chihuahua, México. (No publicada). http://bivir.uacj.mx/ DHI/5e/ProgramaDetallado.asp (2011-06-11).

Vega, Guadalupe (2011). Programas de formación de usuarios en instituciones de educación superior en México. // Reunión Anual del Consejo Nacional para Asuntos Bibliotecarios 23-27, Ciudad del Carmen, México Ciudad del Carmen. (En proceso de publicación).

Vega, Guadalupe; Cortés, Carlos; Quijano, Álvaro. (2006) Diagnóstico de habilidades informativas: propuesta para valorar alumnos de primer ingreso a licenciatura. // Cortés, Jesús; Mears, Berenice (comp.) Implementación de programas de alfabetización informativa: contextos y experiencias: IV Encuentro de Desarrollo de Habilidades Informativas. Ciudad Juárez, Chihuahua, México: Uni- 
versidad Autónoma de Ciudad Juárez; Library Outsourcing, 2006. 99-110.

Vélez García, Adriana Patricia (2005). Uso de las fuentes de información medido a través de las tesis de doctorado de la Facultad de Filosofía y Letras y la Facultad de Ciencias de la UNAM. México: El autor, 2005. Tesis de licenciatura, Universidad Nacional Autónoma de México (UNAM).

Verdugo Sánchez, José Alfredo (1989). Manual para evaluar la satisfacción de usuarios en bibliotecas de institucio- nes de enseñanza superior de la República Mexicana. México: Universidad Nacional Autónoma de México, Centro Universitario de Investigaciones Bibliotecológicas; Asociación Nacional de Universidades e Instituciones de Educación Superior, 1989.

Walsh, Andrew (2009). Information literacy assessment: where do we start? // Journal of Librarianship and Information Science. 41:1 (2009) 19-28.* 